\title{
Classification and Molecular Modeling of Crossbridge Conformations from Tomograms of Insect Flight Muscle.
}

\author{
Kenneth A. Taylor*, Jun Liu*, Gary Connors*, Mary C. Reedy** and Michael K. Reedy** \\ *Institute of Molecular Biophysics, Florida State University, Tallahassee, FL 32306-4380 \\ **Dept. of Cell Biology, Duke University Medical Center, Durham, NC 27702
}

The acquisition of structural information from 3-D images of insect flight muscle (IFM) in various biochemical states requires accurate averages be computed from a near continuum of 3-D structures. The near continuum of structures comes from the fact that there is a mismatch in the axial periods of the two filament systems present in the muscle, the myosin and the actin filaments[1]. Myosin filaments have an axial period of $14.5 \mathrm{~nm}$ whereas the helical repeat of the actin filaments is 77.3 $\mathrm{nm}$. The shortest common period is $232.0 \mathrm{~nm}$, which comprises 16 myosin filament repeats and 3 actin filament repeats. Accurate atomic model building requires that the actin subunits be resolvable in the class averages computed by correspondence analysis.

Earlier work [2, 3], used multireference alignment in combination with hierarchical ascendant classification to generate class averages of the crossbridge motifs, which are regions of the structure containing a $38.7 \mathrm{~nm}$ length of the actin filament and their bound crossbridges. These class averages had a collective resolution of about $7 \mathrm{~nm}$ which is less than that needed to resolve actin monomers in the filaments. To improve the resolution we have tested an alignment procedure that uses just the actin filament, and small portions of the myosin heads, which are windowed from the 3-D image. This actin filament average so generated can be subjected to repeated cycles of alignment and averaging until its signal to noise ratio no longer improves. The aligned motifs are then classified primarily based on the crossbridge features using hierarchical ascendant methods. The procedure, thus, aligns the individual motifs based on the structure that is held in common to each, and then classifies the motifs based on the structure that varies from one motif to the next.

When applied to previously processed motifs from rigor muscle, an improvement in resolution from $7 \mathrm{~nm}$ to $5 \mathrm{~nm}$ was obtained as assessed using the Fourier Shell Correlation (Fig. 1). Using more recently computed tomograms from low dose tilt series from fast frozen, freeze substituted specimens, the resolution improved to between 4.6 and $3.8 \mathrm{~nm}$. The improved specimen preparation and data acquisition procedures contributed to this improvement, but were not wholly responsible because multireference alignment followed by classification failed to resolve the actin monomers in the class averages. In some cases, the new procedure was capable of resolving the $\mathrm{S} 2$ domain of myosin, a structure that consists of an $\alpha$-helical coiled-coil of width $\sim 2 \mathrm{~nm}$ (Fig. 2). This procedure has added benefit for the molecular model building because the actin filament can be built once for all the class averages.

Supported by NIGMS and NIAMSD

[1] A. Miller and R.T. Tregear. J. Mol. Biol. 70 (1972) 85.

[2] H. Winkler and K.A. Taylor. Ultramicroscopy 77 (1999) 141

[3] L.F. Chen et al. J. Struct. Biol., 133 (2001) 221-232 


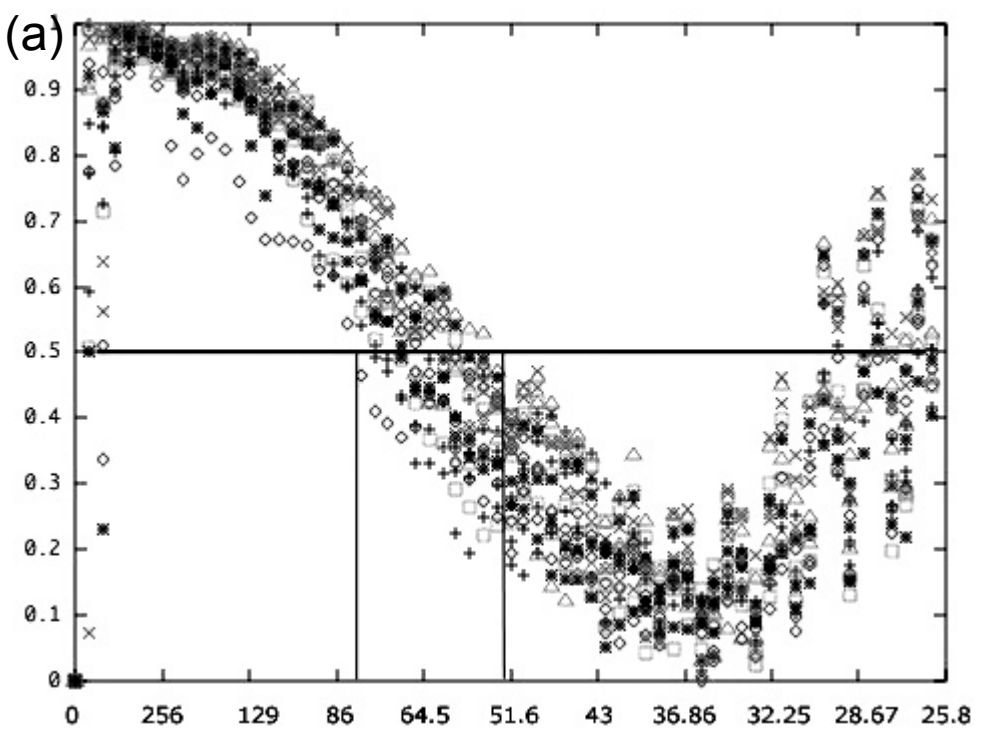

(b)

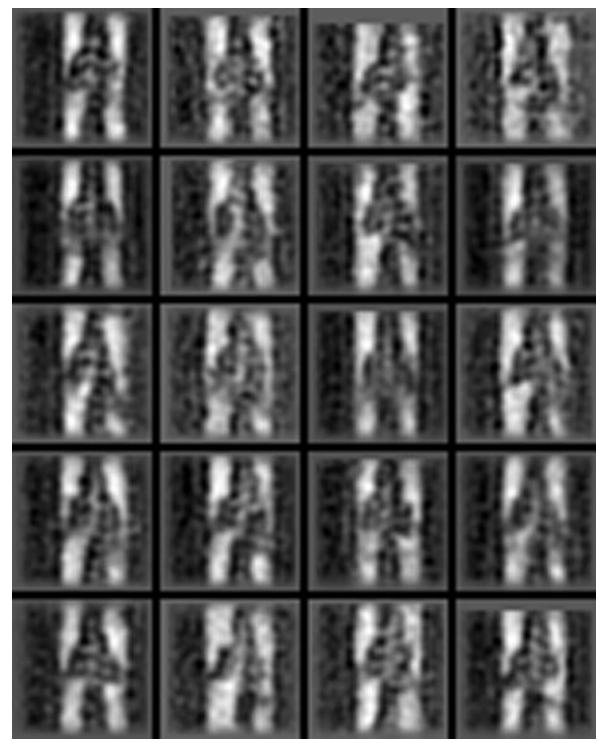

Figure 1. (a) Plot of the Fourier Shell Correlations from previously published data of a tomogram from rigor IFM $[2,3]$. (b) Sections from the class averages showing staggered densities along the actin filament indicating resolution of $\sim 5 \mathrm{~nm}$ in the best class averages.

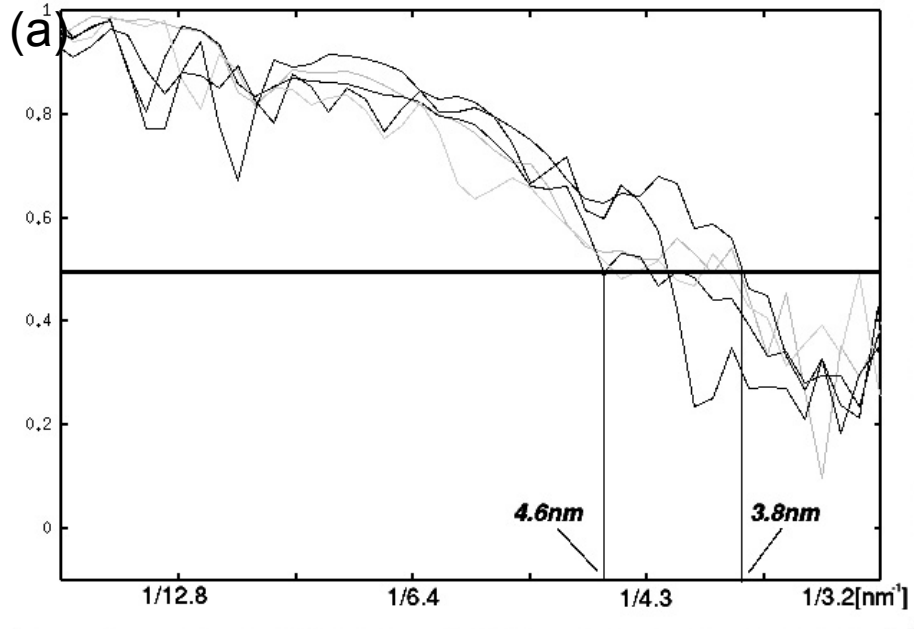

Figure 2. (a) Plot of Fourier Shell Correlations from fast frozen, freeze substituted rigor IFM tomograms. (b) Sections from the center of the 3-D reconstruction. Note the staggered arrangement of density in the actin filament as well as the crossbridges which indicates that the procedure is extracting features at the $5 \mathrm{~nm}$ resolution level.

(b)

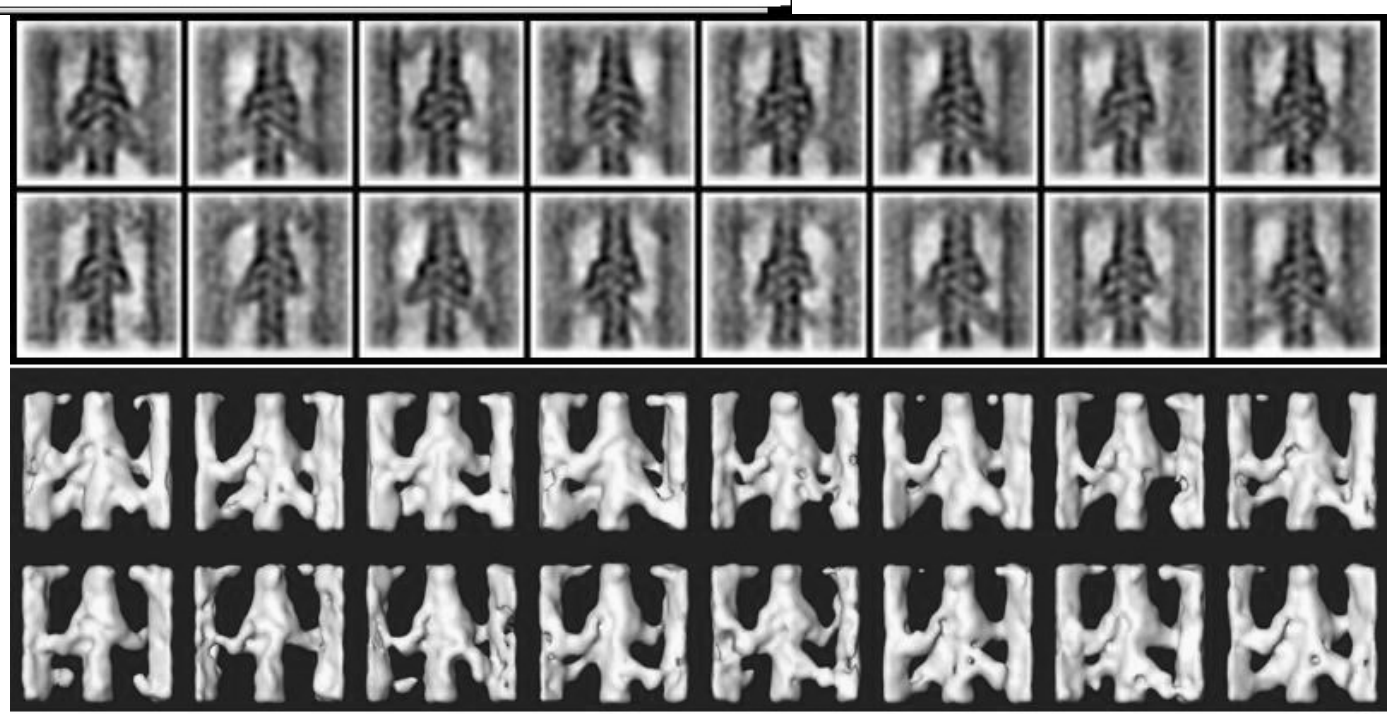

